\section{Sublinguale Immuntherapie: Studien melden Erfolge}

\section{Orale Applikationswege bei der Immuntherapie allergischer Atemwegsreaktionen versprechen eine einfachere Behandlung und eine möglicherweise höhere Compliance als die subkutanen Hyposensibilisierungen. Neue Studien belegen die Wirksamkeit dieser Behandlungsmethode.}

Bisher ließen sich keine eindeutigen Behandlungserfolge mit einer oralen Immuntherapie erzielen, wenn die Antigene sofort geschluckt wurden, ohne sie länger im Mund zu behalten. Pharmakokinetische Studien zeigen jedoch eine Wirksamkeit der sublingualen Applikation, wenn ein Teil der Allergene für längere Zeit im Mund behalten wird.

Daß mit einer sublingualen Immuntherapie eine Verringerung der Allergiesymptome erzielt werden kann, zeigt eine Doppelblindstudie mit 20 Patienten. Die Studienteilnehmer litten an einer durch Hausstaubmilben ausgelösten perennialen Rhinokonjunktivitis. 6 dieser Patienten litten zusätzlich unter leichtem Asthma.

Die Allergiker erhielten 2 Jahre lang entweder eine sublinguale Immuntherapie in Form von Tabletten, die die modifizierten Allergene Dermatophagoides pteronyssinus und Dermatophagoides farinae enthielten, oder Plazebo. Sie mußten die Tabletten unter der Zunge plazieren, 1 bis 2 Minuten lang zum Auflösen im Mund behalten und danach schlucken.

Die Allergendosis wurde in der Aufbauphase bis zu einer Erhaltungsdosis von meist 2000 Allergeneinheiten zweimal pro Woche gesteigert, was der 20fachen Dosis einer sonst üblichen subkutanen Immuntherapie entspricht.

In der täglich aufgezeichneten Beurteilung der Symptome der Rhinokonjunktivitis durch die Patienten zeigte sich, daß in der mit Verum behandelten Gruppe signifikant weniger Beschwerden als in der Plazebogruppe auftraten. Am besten zu beobachten war dies während der Wintermonate, in denen die Allergenbelastung der Patienten durch Hausstaubmilben am höchsten ist.

\section{Auch bei Respondern: \\ Entzündung persistiert minimal}

Auch Messungen von objektiven Parametern der allergischen Reaktion ließen auf einen Behandlungserfolg schließen. So nahm beispielsweise nach Allergenexposition an der Bindehaut zu Beginn der Behandlung, nach einem und nach 2 Jahren die Infiltrationsrate der inflammatorischen Zellen ab. Auch die Zahl der interzellulären Adhäsionsmoleküle 1 (ICAM-1) reduzierte sich im ersten Behandlungsjahr signifikant.

Eine minimale persistierende Entzündung trat auch bei symptomfreien Patienten auf, da Neutrophile schon vor der Allergenexposition in der Bindehaut vorhanden waren. Die Zahl der neutrophilen Granulozyten nahm jedoch während der Immuntherapie ab, nicht jedoch unter Plazebogabe. Weiterhin konnte eine Reduktion der Serumkonzentration des eosinophilen kationischen Proteins festgestellt werden.

(jn)

Passalacqua G, et al.: Randomized controlled trial of local allergoid immunotherapy on allergic inflammation in mite-induced rhinoconjunctivitis. Lancet 1998; 351: 629-32. - Holt PG sease. Lancet 1998; 351: 613

\section{Sublinguale Immuntherapie: Was ist gesichert?}

Bei der sublingualen Immuntherapie wird ein spezifisches Allergen in ansteigender Dosierung peroral oder sublingual appliziert. Doch besteht über die reproduzierbare Wirksamkeit dieser Methode immer noch keine Einigkeit. So wurde in einigen Untersuchungen ein therapeutischer Effekt nachgewiesen, in anderen wiederum nicht. Neuere, kontrolliert durchgeführte Studien, bei denen im Vergleich zur subkutanen Therapie höhere Allergendosen gegeben wurden, belegen jedoch eine Wirksamkeit.

Obwohl in diesen Untersuchungen keine erhöhte Nebenwirkungsrate gefunden wurde, wird immer wieder die Sicherheit dieser Therapiemethode in Frage gestellt: Immerhin findet die Medikation nicht in Gegenwart eines Arztes statt, so daß der Patient beispielsweise falsch dosieren kann. Doch erfolgt die tropfenweise Steigerung der Allergenmenge im Vergleich zur subkutanen Injektion wesentlich langsamer, wodurch die Gefahr plötzlich auftretender Unverträglichkeiten herabgesetzt ist.

Derzeit kann die sublinguale oder perorale Hyposensibilisierung allerdings noch nicht als gleichwertig mit der klassischen Hyposensibilisierung angesehen werden.

Sollte die Zahl der gut dokumentierten Studien jedoch noch weiter zunehmen, dürfte diese Behandlungsmethode in Zukunft einen höheren Stellenwert im Rahmen der Therapiemöglichkeiten allergischer Erkrankungen - insbesondere bei Kindern einnehmen.

(red) 